\title{
Calculation of the influence of slot geometry on the magnetic flux density of the air gap of electrical machines: three-dimensional study
}

\author{
Rodrigo A. Lima, A. C. Paulo Coimbra, Tony Almeida, Viviane Margarida Gomes, \\ Thiago M. Pereira, Aylton J. Alves and Wesley Pacheco Calixto
}

\begin{abstract}
The objective of this work is to investigate the influence of slotted air gap constructive parameters on magnetic flux density of rotating machines. For this purpose, different approaches were used to solve the air gap field diagram using finite element method and the magnetic field distribution uniformity was evaluated by Carter's factor calculation on twodimensional and three-dimensional models. Sensitivity analysis of slot constructive parameters was performed and results show that slot geometry modifies the magnetic flux on air gap and shifts the air gap magnetic equipotential midline of double slotted machines. Finally, minimization of Carter's factor on twodimensional model presents an optimized slot geometry with a near uniform magnetic flux density distribution.

Index Terms - Carter's factor, finite elements method, rotating machines.
\end{abstract}

\section{INTRODUCTION}

$\mathrm{M}$ AGNETIC circuits of rotating machines are briefly composed by its ferromagnetic parts (rotor and stator) and air gap [1]. Thus, analytic equations and numerical method simulation are both used on magnetic circuit design process to determine parameters such as torque and machine excitation current [2], [3]. Magnetic flux distribution on air gap has great influence on machine performance because the most part of magnetic energy distribution is contained in air gap domain [1], [4]. Once there are magnetic flux density fluctuations in air gap domain due the presence of slots in ferromagnetic parts, air gap reluctance is dependent of the rotor and stator relative position [5], [6].

This work was supported by the Coordination for the Improvement of Higher Education Personnel-CAPES-PDSE Process number: 99999.003605/2014-00, National Council of Scientific and Technologic Development of Brazil $-\mathrm{CNPq}$ and Research Support Foundation of Goiás State-FAPEG.

R. A. Lima (corresponding author), T. M. Pereira and W. P. Calixto are with Electrical and Computer Engineering School, Federal University of Goias, UFG, Av. Universitaria, 1488 Qd. 86 Bl. A Zip 74605-010, Goiania, Goias, Brazil and with Experimental \& Technological Research and Study Group, NExT of Federal Insitute of Goias, IFG, Rua 75, 46, Centro, Zip: 74055-110, Goiania, Goias, Brazil (email: rodrigo.lima@,ifg.edu.br, thiago.pereira@ifg.edu.br, wpcalixto@gmail.com ).

A. C. P. Coimbra and T. Almeida are with Institute of Systems and Robotics, ISR of Coimbra University, UC, Rua Silvio Lima, Zip 3030-194, Coimbra, Portugal (email: acoimbra@deec.uc.pt, tony@deec.uc.pt).
V.M. Gomes and A. J. Alves are with Experimental \& Technological Research and Study Group, NEXT - IFG (email: vivianemargarida@gmail.com, aylton.alves@ifg.edu.br ).

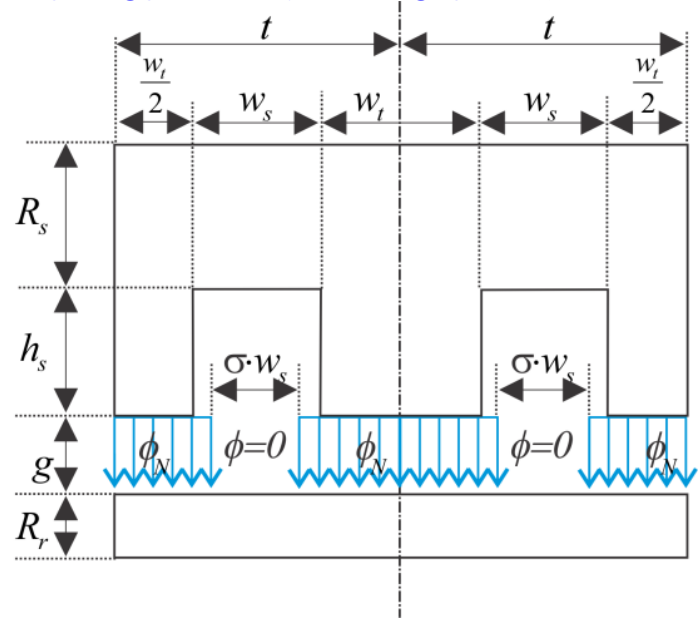

Fig. 1. Simplified air gap of a machine with slots on stator's surface, where, $t$ is stator tooth pitch, $w_{t} / w_{s}$ tooth/slot width, $h_{s}$ stator slot height and $R_{s} / R_{r}$ stator/rotor yoke height. Blue lines represent uniform magnetic flux, $\phi_{N}$.

Although analytic description for magnetic flux density in air gap domain is not an easy task, F. W. Carter presented an analytic equation to quantify the magnetic flux reduction in slotted air gap by introducing the concept of equivalent air gap [7]- [9]. Carter considered that magnetic flux reduction is equivalent to replace the slotted air gap with length $g$ for an equivalent smooth air gap with length $g_{e q}=k_{c} \cdot g\left(k_{c} \geq 1\right)$. The term $k_{c}$ is called Carter's factor and its value is influenced by air gap magnetic flux distribution, where $k_{c}=1$ is equivalent to a uniform magnetic flux distribution.

The purpose of this paper is to present a study of slot geometry influence on magnetic flux density distribution in air gap dominion using Carter's factor to evaluate the flux distribution uniformity. The finite element method (FEM) was used to solve magnetic flux distribution in air gap domain in two and three dimensional models and different approaches to evaluate Carter's factor were compared. Additionally, it was performed a sensitivity analysis to study the influence of air gap constructive parameters influence on Carter's factor 
considering two different slot patterns. Finally, double-slotted air gap geometry effect was considered on a case study where different slot patterns were combined.

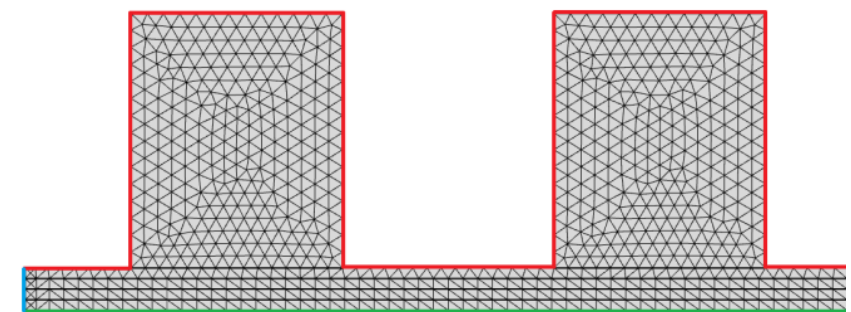

Fig. 2. Discretized domain representation of air gap using FEM. Red boundaries and green boundaries represents Dirichlet's known potential and blue boundaries represents Neumann boundary condition.

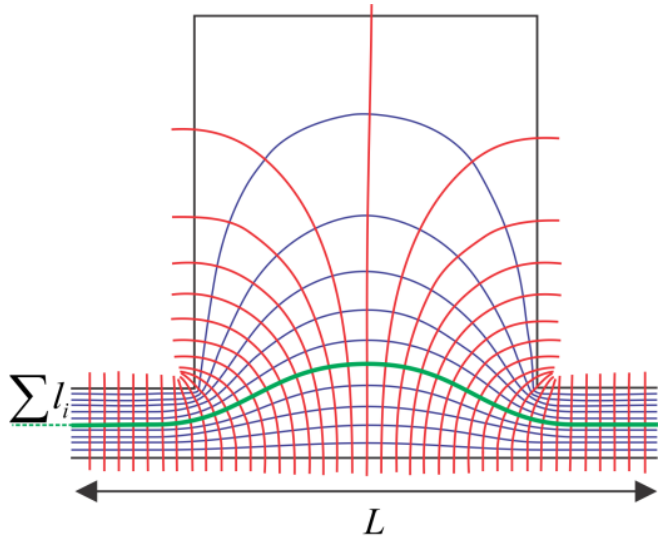

Fig. 3. Magnetic flux diagram determined by FEM in air gap domain with length $L$. Green line represents intermediate magnetic equipotential line with length $\sum l_{i}$ numerically determined.

\section{CARTER'S FACTOR}

Carter's original study considers a simplified air gap geometry to quantify the magnetic flux reduction by the slot presence. Fig. 1 presents the constructive relations considered in air gap.

To describe the magnetic flux density in air gap domain, Carter considered that regions close to teeth have uniform magnetic flux (blue arrows in Fig. 1) while regions near of slot opening have null magnetic flux. Null flux regions will reduce the mean magnetic flux in air gap domain and the Carter's factor is defined by [1], [4], [7].

$$
k_{c}=\frac{t}{t-\sigma w_{s}} \text {. }
$$

The value of $\sigma$ on (1) depends on air gap constructive parameters and is related to the magnetic flux distribution near the teeth. Carter found (2) using Schwartz-Christoffel's conformal mapping on a simplified slot geometry [7]. Other methodologies may be found in literature to determine $\sigma$. However, this work will consider the empirical expression (3) proposed by Langsdorff for comparison of analytic results [10].

$$
\sigma=\frac{2}{\pi}\left\{\arctan \left(\frac{w_{s}}{2 g}\right)-\frac{g}{w_{s}} \ln \left[1+\left(\frac{w_{s}}{2 g}\right)^{2}\right]\right\}
$$

$\sigma=\frac{w_{s} / g}{5+w_{s} / g}$

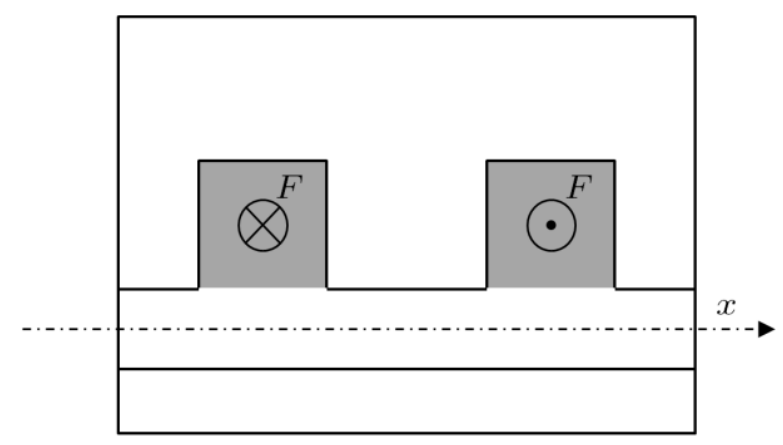

(a)

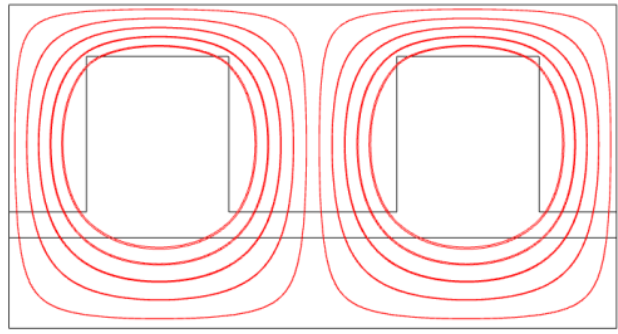

(b)

Fig. 4. (a) Domain representation and current density considered. (b) Flux diagram determined by FEM.

On double slotted air gaps, the Carter's factor is defined by the product of the rotor Carter's factor, $k_{c r}$, and the stator Carter's factor, $k_{c s}$. In this case, $k_{c r}$ and $k_{c s}$ are independently calculated using (1) and $\sigma$ is determined by equation (2) or (3) [1], [5], [6].

$k_{c}=k_{c r} \cdot k_{c s}$

Although many authors consider sufficient in practice, the results obtained by expressions (1), (2), (3) and (4) are not accurate [11]- [15]. More accurate methods involve air gap magnetic field diagram solution by numerical methods. In this work the finite element method is applied on different approaches for magnetic field diagram solution in air gap domain.

The first approach, using the field diagram solution, considers that neither electric charges nor currents exist within the air gap domain. In this case, the problem is totally characterized by setting magnetic scalar potential $V_{m}$.

Magnetic induction lines are mapped by solving two dimensional Laplace's equation with respective Neumann's and Dirichlet's boundaries conditions on analogy to electrostatic behavior [1], [6], [13]. Fig. 2 illustrates Neumann's boundary conditions (blue and green lines), and red regions represent the known potential of Dirichlet condition applied to the rotor and the stator surface.

Fig. 3 illustrates the magnetic equipotential lines mapping (blue lines) and the magnetic flux density $\vec{B}$ (red lines). The intermediate equipotential line obtained in diagram has greater length than intermediate equipotential line of a smooth air gap. 
The intermediate equipotential line length is given by $\sum l_{i}$ and is determined numerically [13]. The ratio of the actual intermediate equipotential line length $\sum l_{i}$ by theoretical smooth air gap length $L$ calculates Carter's factor [16], [17].

$k_{c}=\frac{\sum l_{i}}{L}$

Another Carter's factor definition using FEM is the ratio between magnetic flux density peak value in air gap, $B_{\max }$, and the magnetic flux density's average along the stator pitch [1], [14].

$k_{c}=\frac{B_{\max }}{B_{a v}}$

where $B_{a v}$ is the average value of magnetic flux density along the tooth pitch axis $x$, given by [1], [14],

$B_{a v}=\frac{1}{2 t} \int_{0}^{2 t} B(x) d x$

The second approach for the air gap field diagram calculation considers the electric current in the slots windings and maps the induction lines considering both air gap and ferromagnetic parts of the machine. Fig. 4a indicates the direction of current density ( $F$ ) of each winding. The result is shown in Fig. 4b and since this approach do not considers equipotential lines, Carter's factor is determined only by (6) and (7).

Although results obtained by two-dimensional magnetic field mapping provides more accurate results than analytical method they don't describes the dynamical behavior of rotating machines. On double slotted air gaps the magnetic flux depends on relative position of rotor and stator slots. The squirrel cage rotor has an angular deviation of one slot step to reduce the magnetomotive force loss in machine. However, the angular deviation of rotor breaks the axial symmetry and two-dimensional approximation of Laplace's equation are not possible because magnetic scalar potential and magnetic flux density has axial dependency.

This paper proposes a three-dimensional analysis of magnetic field diagram in airgap domain and the generalization of equations (5), (6) and (7) for Carter's factor calculation. For magnetic scalar potential approach, the equation (6) can be extended to three-dimensional domain taking into consideration magnetic equipotential surfaces in place of magnetic scalar equipotential lines of twodimensional approach.

$\mathrm{k}_{c}=\frac{A_{m e f}}{A_{g}}$

where, $A_{\text {mef }}$ is the intermediate magnetic equipotential surface numerically determined in airgap domain and $A_{g}$ is the theoretical intermediate magnetic potential of the smooth air gap.

The three-dimensional evaluation of Carter's factor by (8) is equivalent to the mean of the results of (5) taken into infinitesimal angular displacements in the one slot step interval. In addition, three-dimensional generalization of excitation current approach leads to the modification of the calculus of the mean magnetic field density in axial

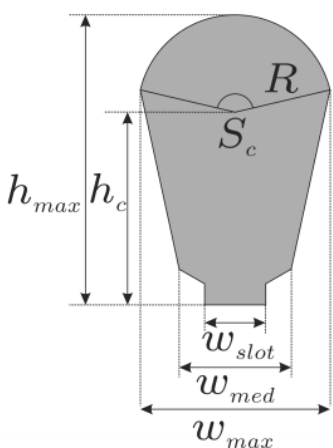

(a)

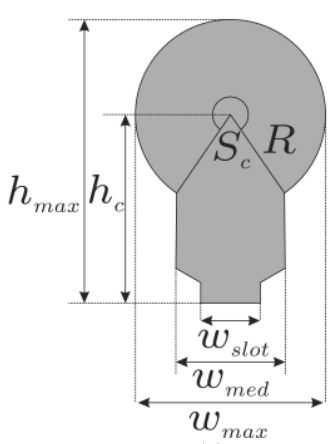

(c)

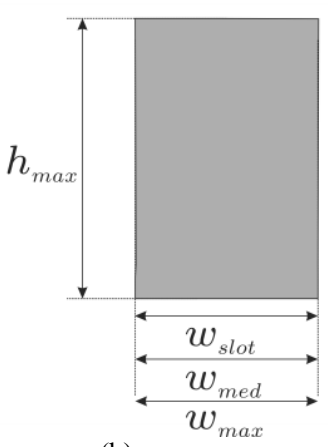

(b)

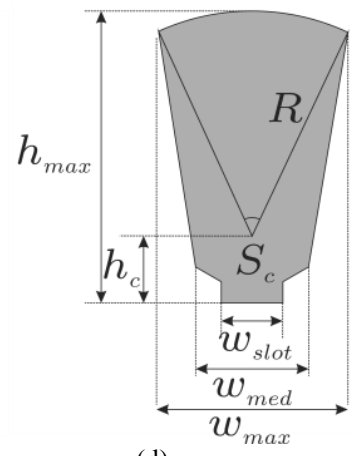

(d)
Fig. 5. (a) Generic slot representation and main parameters to be varied $S c=120^{\circ}$. (b) Resulting geometry for $w_{\text {slot }}=w_{\text {med }}=w_{\max }$ and $R \rightarrow \infty$. (c) $R=w_{\max }$ and $S c=270^{\circ}$ (Pattern I). (d) $S c=60^{\circ}$ and $R \geq w_{\max }($ Pattern II).

TABLE I

SLOT CONSTRUCTIVE INITIAL VALUES

\begin{tabular}{cccc}
\hline \hline$h_{\max }(\mathrm{mm})$ & $w_{\max }(\mathrm{mm})$ & $w_{\text {slot }}(\mathrm{mm})$ & $t(\mathrm{~mm})$ \\
\hline 12 & 6 & 2 & 10 \\
\hline \hline
\end{tabular}

coordinates

$B_{a v}=\frac{1}{A_{g}} \iint B\left(r_{\text {mean }}, \theta, z\right) d \theta d z$

where, $r_{\text {mean }}$ the axial coordinate equivalent to the radius coordinate of minimum axial distance between rotor and stator teeth. The maximum value of magnetic flux density $B_{\max }$ is determined on axial coordinate $r_{\text {mean }}$ and the Carter's factor is then calculated by (6).

\section{Methodology}

A generic slot was created to study the geometry effect in air gap magnetic flux. Changing its main constructive parameters, it is possible to observe changes in slot pattern. In Fig. 5 are presented some possible changes in the slot geometry. The variation of parameters $h_{\max }, w_{\max }, R, w_{\text {slot }}$, $w_{\text {med }}$ and $S_{c}$ creates new air gaps patterns.

Thus, the field diagram is solved for magnetic potential approach and magnetizing current approach. Carter's factor 
then quantifies flux's uniformity [1], [13], [14]. A small induction machine stator inspired the initial values of the slot. The slots constructive parameters are shown in Table I.

Values of $h_{\max }$ and $w_{\max }$ are fixed as indicated in Table I to ensure that the winding will be accommodated. The variation of the parameters $R$ and $S_{c}$ is divided in two slots patterns. First pattern has $R$ and $S_{c}$ relations given by (10a) and (10b) and is illustrated in Fig. $5 \mathrm{c}$.

$R=w_{\text {max }}$
$S_{c}=\left[180^{\circ}, 2 \cdot \arcsin \left(\frac{w_{\text {slot }}}{2 R}\right)\right]$

Second pattern is illustrated in Fig. 5d and the parameters $R$ and $S_{c}$ are given by (11a) and (11b), respectively.

$R=\left[w_{\max }, h_{\max }\right]$

$S c=2 \cdot \arcsin \left(\frac{w_{\max }}{2 R}\right)$

Both patterns relate slot opening $w_{\text {slot }}$ and intermediate opening $w_{\text {med }}$ by (12a) and Eq.(12b).

$w_{\text {slot }}=\left[w_{\min }, w_{\max }\right]$

$w_{\text {med }}=\frac{7 w_{\max } \cdot w_{\min }}{3\left(w_{\max }-w_{\min }\right)}+\frac{3 w_{\max }-10 w_{\min }}{3\left(w_{\max }-w_{\min }\right)} \cdot w_{\text {slot }}$

where (12b) came from slot geometrical analysis for $w_{\text {med }}=w_{\text {slot }}$ when $w_{\text {slot }}=w_{\max }$. In (12a) was adopted $w_{\min }=1$ $\mathrm{mm}$. The viable space is represented by equations (10b), (10a) and (12a).

Five case studies analyses the effect of air gap variation using both magnetic field mapping approaches. The parameters variation and domain discretization are defined by (11) and (12) and resulted in the sensitivity study of the construction parameters [18].

The sensitivity analysis study is based on the factorial experiment design. For textual comprehension, 'factor' in the factorial experiment design is related to the decisions variables considered in sensitivity study. On this paper, Carter's factor is considered the system response or experimental result of the factorial experimental design. The factorial experiment design takes on all possible level combinations of decisions variables and schematically organizes their combinations in a planning diagram. The planning diagram relates the decision variables combined levels with their respective system response. Considering two generic variables $P$ and $Q$, with two discrete levels, the experiment is called $2^{2}$ factorial design and has four system response $Y_{j}$. In this case, the planning diagram can be picture by Fig. 6 .

The principal effect of decisions variables is given by,

$\Pi_{P}=\frac{Y_{2}+Y_{4}}{2}-\frac{Y_{1}+Y_{3}}{2}$

$\Pi_{Q}=\frac{Y_{3}+Y_{4}}{2}-\frac{Y_{1}+Y_{2}}{2}$
The respective sensitivity analysis of $P$ and $Q$ considers the mean of different $2^{2}$ factorial design principal effects

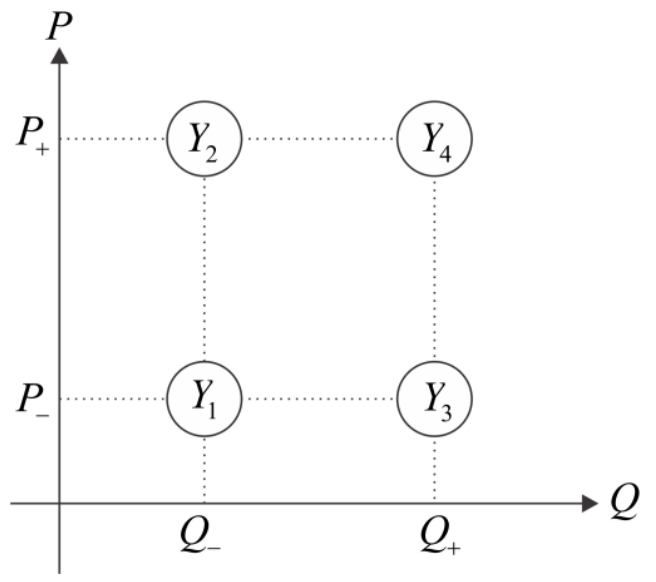

Fig. 6. System response diagram for a $2^{2}$ factorial experiment design.

calculated by (13) and is expressed by its relative value. The respective mean relative percentage value is denoted by Sens $_{P}$ and Sens $_{Q}$, respectively for $P$ and $Q$.

A. Case I:

The first case study is a sensitivity analysis on $R, S_{c}$ and $w_{\text {slot }}$. In this case, air gap magnetic equipotential lines are mapped applying the scalar magnetic potential formalism $V_{m}$ and Carter's factor is determined by (5) [1], [13], [14]. The sensitivity analysis is then performed by taking a discretized domain from equations (10) and (11). For slot pattern I, the principal effect of $S_{c}$ and $w_{\text {slot }}$ are evaluated using (13) and the sensitivity of each parameter is calculated as the average of several $2^{2}$ factorial design in distinct two levels domains. Analogously, the sensitivity analysis of $R$ and $w_{\text {slot }}$ is performed to slot pattern II.

Additionally, this case study, magnetic flux density lines are mapped on the air gap domain. The maximum magnetic flux density $B_{\max }$ and mean magnetic flux density $B_{a v}$ by FEM and the Carter's factor is then evaluated using (6). The sensitivity analysis study is then performed.

\section{B. Case II}

The sensitivity analysis on $R, S_{c}$ and $w_{\text {slot }}$ for air gap magnetic flux setting the winding current. Carter's factor is determined by (6) [1], [13], [14]. Althought the winding current approach is also a FEM method to determine magnetic flux diagram is not possible determine scalar magnetic equipotential curves and Carter's factor determination from (5). The sensitivity analysis is then performed only to the excitation current approach by the calculation of the respective principal effects of decision variables in the discretized domain in analogous form of Case I.

\section{Case III:}

This case study shows that air-gap equipotential midline, 
defined by the magnetic equipotential line with minor influence by the air gap geometry, is not always located in the middle of the airgap. Different geometries of double slotted air

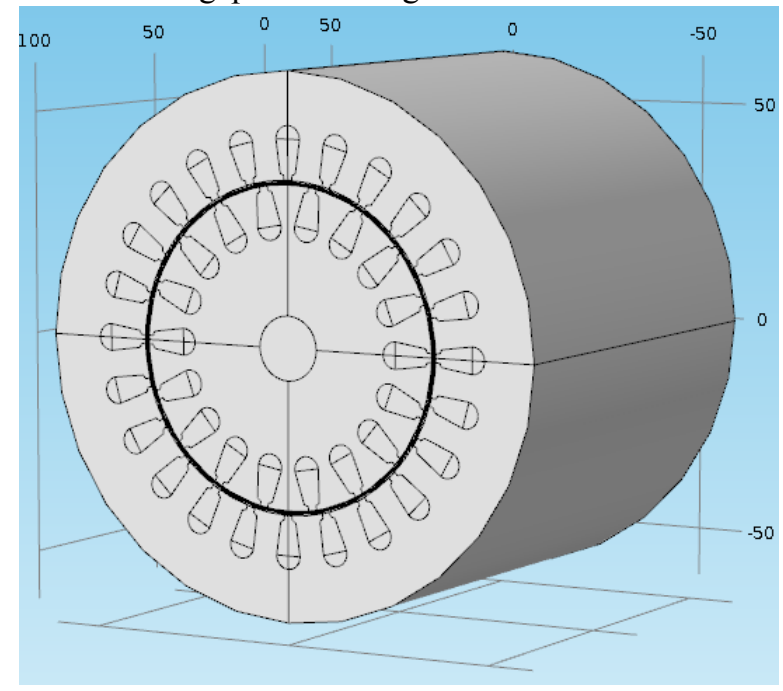

(a)

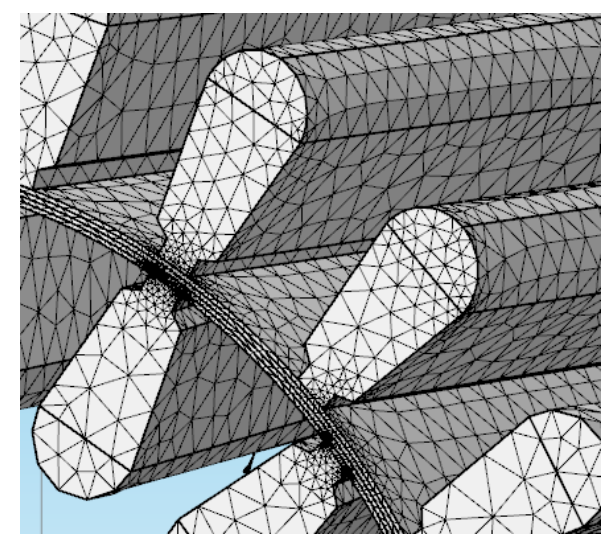

(b)

Fig. 7 (a) Three-dimensional model of double slotted airgap. (b) Detailed view of finite elements mesh on airgap domain.

gaps were considered and the results show that magnetic equipotential middle line position approach to the rotor surface or stator surface [16], [17].

\section{Case IV}

On this case study an air gap geometry is proposed by using constrained optimization of Carter's factor by real-coded genetic algorithm [19]. This case study considers the minimization problem

$\min k_{c}$

$\vec{x} \in S 2$

where $\vec{x}=\left(R, S_{c}, w_{\text {slot }}, w_{\text {med }}\right)$ is the decision variables vector, and $\Omega$ is the viable space defined by equations (10), (11) and (12). On an optimization process, each decision vector $\vec{x} \in \Omega$ is an individual belonging to a finite population that evolves by crossover and mutation agents on a natural selection scheme inspired by Darwin evolution's theory. The population evolves by successive iterations called generations until an individual satisfies a determined stop criteria.

On this case study a population of 50 individuals evolves until the error defined by $\varepsilon=k_{c}-1$ has a value minor to $10^{-3}$ or the number of iteration achieves 100 generations. The Carter's factor is calculated using magnetic field diagram by FEM and (6). On the first geometry proposed, the constructive parameters on Table I are fixed and the optimization process takes into consideration only the parameters $R$ and $S_{c}$. The objective of this study is to compare the Carter's factor of an optimized geometry with the original geometry from the real induction machine. On the second geometry proposal, (14) is solved to $\vec{x} \in \Omega$. On both geometries, the Carter's factor obtained by FEM is compared to analytical calculations by (1), (2) and (3).

\section{E. Case $V$}

The final case study uses a generic three-dimensional model of a double slotted air-gap created in FEM. The air gap constructive parameters were parametrized with initial values given in Table I. Using magnetic scalar equipotential approach the magnetic field is mapped the intermediate equipotential surface are numerically determined. The Carter's factor for the theoretical double slotted air gap is then evaluated by (8) and compared with the results evaluated by the analytical approach.

Additionally, the effect of the rotor twist angle, $\theta_{\text {rot }}$, analyzed varying the value of $\theta_{\text {rot }}$ from $5^{\circ}$ to $40^{\circ}$. The Fig. $7 \mathrm{a}$ represents the generic air gap model and the detailed view of finite elements mesh is depicted on Fig. 7b. The air gap region was divided on four layers to increase the number of finite elements on the air gap domain.

\section{RESULTS}

\section{A. Case I}

Sensibility analysis of Carter's factor due to slot geometrical parameters variation was performed calculating the relative deviation of $k_{c}$ by an associated perturbation on parameters viable space. Carter's factor was calculated by (5) using magnetic scalar potential mapping by FEM. Results for slot pattern I are presented in the first and second columns of Table II, where Sens $S_{S c}$ and Sens ${ }_{w s l o t}$ are the respective mean sensibility of $S_{c}$ and $w_{\text {slot }}$ parameters taken by the $2^{n}$ factorial combination of perturbations in viable space. Additionally, a relative deviation in $S_{c}$ with respect the initial values of Table I and their respective relative deviation on $k_{c}$ is depicted in Fig. 8.

TABLE II

CASE STUdy I - MAGNETIC POTENTIAL APPROACH

Pattern I

Pattern II

\begin{tabular}{rccc}
\hline \hline Sens $_{S c}$ & Sens $_{\text {wslot }}$ & Sens $_{R}$ & Sens $_{\text {wslot }}$ \\
\hline $2.013 \%$ & $97.987 \%$ & $0.075 \%$ & $99.925 \%$ \\
\hline \hline
\end{tabular}


On the other hand, sensitivity analysis of $R$ and $w_{\text {slot }}$ parameters to slot pattern II are presented in third and fourth columns of Table II. It is possible to observe that in both

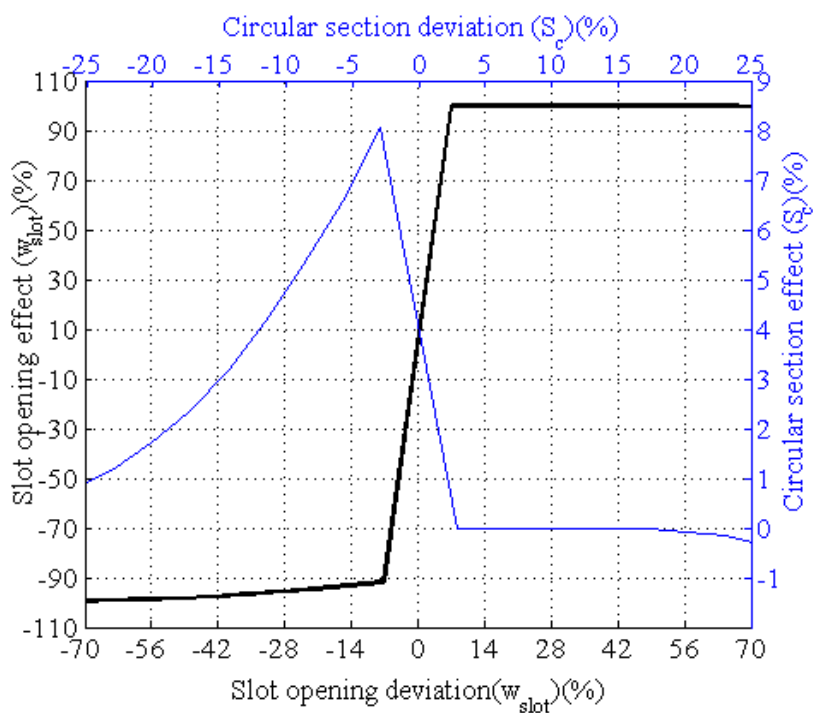

Fig. 8. Sensitivity analysis of $S_{c}$ parameter (blue axis) and sensitivity analysis of $w_{\text {slot }}$ parameter (black axis) for slot pattern I by magnetic scalar potential calculation of $k_{c}$ by (5).

patterns the major influence for $k_{c}$ provided by $w_{\text {slot }}$ parameter, although in slot pattern I $S_{c}$ has significant contribution when compared with $R$ parameter in slot pattern II.

After the magnetic scalar potential analysis, the magnetic field density was evaluated in the air gap tooth axis and Carter's factor is determined by (6). Sensitivity analysis of Carter's factor using the magnetic field density approach was carried out analogously to analysis performed for the magnetic scalar potential approach. Table III presents the respective sensitivity of $S_{c}$ and $w_{\text {slot }}$ for slot pattern I in the first and second columns. Additionally, the sensitivity of $R$ and $w_{\text {slot }}$ parameters for slot pattern II are presented in third and fourth columns of Table III.

TABLE III

CASE STUDY I - MAGNETIC FIELD DENSITY APPROACH

\begin{tabular}{crcc}
\hline \hline \multicolumn{2}{c}{ Pattern I } & \multicolumn{2}{c}{ Pattern II } \\
\hline \hline Sens $_{S c}$ & Sens $_{\text {wslot }}$ & Sens $_{R}$ & Sens $_{\text {wslot }}$ \\
\hline $0.3 \%$ & $99.7 \%$ & $0.008 \%$ & $99.992 \%$ \\
\hline \hline
\end{tabular}

\section{B. Case II}

Magnetic field density determination uses FEM and excitation current approach in the tooth axis of air gap. Because the magnetic field is directly determined on this method, Carter's factor is determined only by (6). Following the same methodology described in Case I, sensitivity analysis was performed for both slot patterns considering relative perturbations in their respective viable spaces and calculating the resulting relative deviation in $k_{c}$. Sensitivity of $S_{c}$ and $w_{\text {slot }}$ for slot pattern I are presented in the first and second
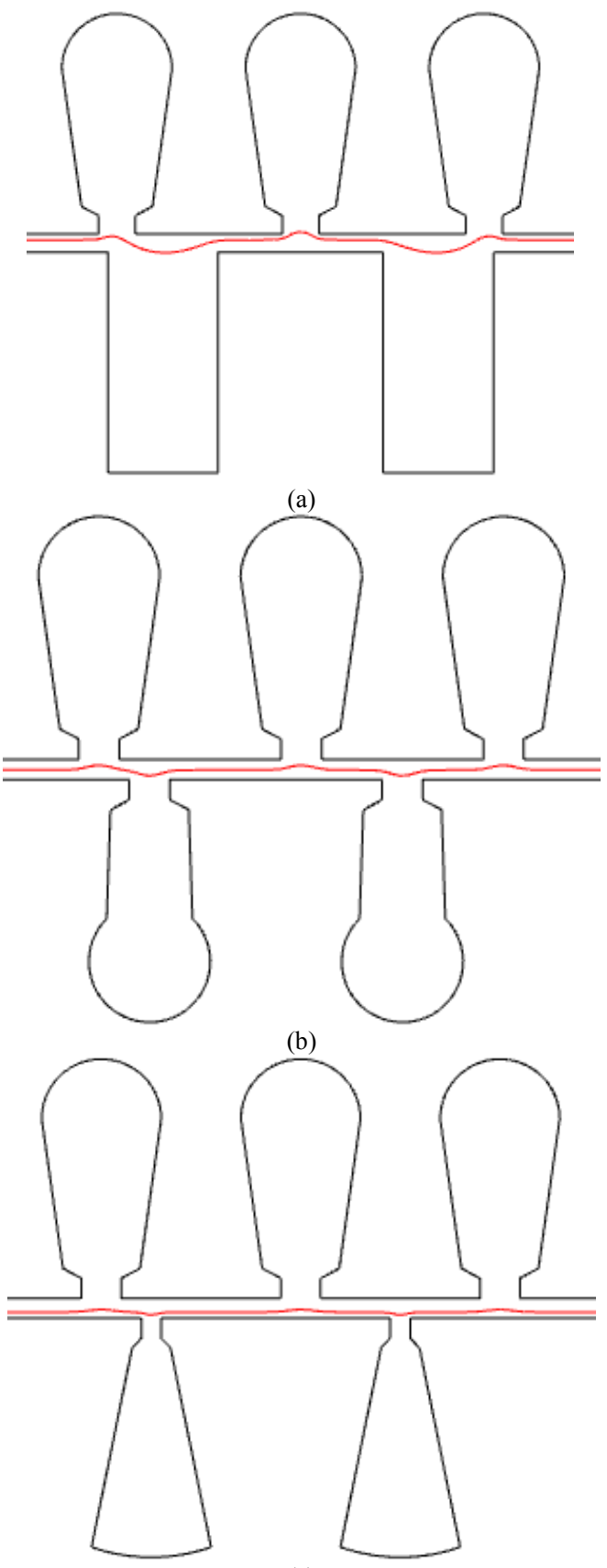

(c)

Fig. 9. Double slotted air-gaps patterns and their respective equipotential midline (red line). (a) Slots A and B, (b) slots A and C, (c) slots A and D.

columns of Table IV. The sensitivity values of $R$ and $w_{\text {slot }}$ are presented in the third and fourth columns of Table IV. 
TABLE IV

CASE STUDY II

\begin{tabular}{cccc}
\hline \hline \multicolumn{2}{c}{ Pattern I } & \multicolumn{2}{c}{ Pattern II } \\
\hline \hline Sens $_{S c}$ & Sens $_{\text {wslot }}$ & Sens $_{R}$ & Sens $_{\text {wslot }}$ \\
\hline $0.26 \%$ & $99.74 \%$ & $0.02 \%$ & $99.98 \%$ \\
\hline \hline
\end{tabular}

\section{Case III}

This section presents the analysis of double air gap geometry influence in air gap magnetic equipotential midline position. Equipotential midline is defined by the equipotential line that have minor influence by slot's presence in air gap ferromagnetic surfaces. Due to space limitations, the four slot patterns illustrated in Fig.9 were combined to form only three different types of air gap geometries. Table $\mathrm{V}$ summarizes the slots constructive parameters.

TABLE V

SLOT PATTERN

\begin{tabular}{cccc}
\hline \hline Pattern & $w_{\text {slot }}(\mathrm{mm})$ & $R(\mathrm{~mm})$ & $S_{c}(\mathrm{deg})$ \\
\hline A & 2 & 3 & 180 \\
B & 6 & - & - \\
C & 2 & 3 & 270 \\
D & 1 & 9 & 19.47 \\
\hline \hline
\end{tabular}

The red line depicted in Fig. 9 represents the equipotential midline mapped by FEM in air gap domain. Table VI presents the respective axial position of midline and the relative deviation from theoretical position. Axial position $y_{\text {line }}$ is measured from the rotor surface to the equipotential midline's unperturbed region and is presented in second column of Table VI. Third column shows the deviation $\delta_{\text {line }}$ from the theoretical position by relative to the total size of the air gap.

TABLE VI

MIDLINE POSITION AND DISPLACEMENT

\begin{tabular}{ccc}
\hline \hline Pattern & $y_{\text {line }}(\mathrm{mm})$ & $\delta_{\text {line }}(\mathrm{mm})$ \\
\hline A \& B & 0.68 & 18 \\
A \& C & 0.47 & 3 \\
A \& D & 0.30 & 20 \\
\hline \hline
\end{tabular}

\section{Case IV}

Optimized slot geometries are presented in Fig. 10. The optimization of slot constructive parameters results in an error of $\varepsilon=1 \cdot 10^{-6}$ and is equivalent to a uniform magnetic field flux on air gap. Table VII summarizes slot constructive parameters and compares their respective Carter's Factor by FEM and analytical methods.

The results of Carter's factor calculation in different methodologies show that the proposed slot geometry have improved the simulated magnetic field uniformity. However, only the FEM methodology can determine accurately the contribution of $R$ and $S_{c}$ parameters.

\section{E. Case V}

On this case study the magnetic field mapping of the geometry depicted in Fig.7a with parameters indicated by Table I result in an intermediate equipotential surface. The total area of the equipotential was numerically determined by FEM. Table VIII presents the comparison of Carter factor evaluated to the three-dimensional air gap methodology (3DFEM) and the analytical equations presented by Carter and Langsdorff.

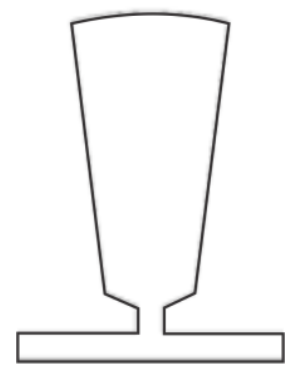

Fig. 10. Optimized slot geometry by genetic algorithm and Carter's factor calculation by FEM.

TABLE VII

SLOT CONSTRUCTIVE PARAMETERS AND CARTER'S FACTOR COMPARISON

\begin{tabular}{ccc}
\hline \hline Parameter & Original & Optimized \\
\hline$R$ & $3.00 \mathrm{~mm}$ & $11.87 \mathrm{~mm}$ \\
$w_{\text {slot }}$ & $2.00 \mathrm{~mm}$ & $1.00 \mathrm{~mm}$ \\
$S_{c}$ & $180.00^{\circ}$ & $29.28^{\circ}$ \\
$w_{\text {med }}$ & $3.89 \mathrm{~mm}$ & $3.33 \mathrm{~mm}$ \\
$k_{c}(6)$ & 1.062 & 1.000 \\
$k_{c}(1)$ and (2) & 1.057 & 1.017 \\
$k_{c}(1)$ and (3) & 1.060 & 1.015 \\
\hline \hline
\end{tabular}

The results evaluated by the 3D-FEM methodology show little divergence to the analytical methodology. The reason for the divergence on the values of Carter's factor can be explained by the magnetic field mapping in the air gap domain. The twist angle of one slot step allow to evaluate different relative positions between the stator and rotor slots. The resulting intermediate magnetic scalar equipotential surface also represents the dynamical character of the magnetic flux uniformity on this case, once the equipotential surface area is conserved with the angular displacement of the rotor.

The effect of twist angle is depicted on the Fig. 11, where an 18 slots rotor is represented with twist angles of $5^{\circ}, 20^{\circ}$ and $40^{\circ}$, respectively. Usually the value of $\theta_{r o t}$ is equivalent to one slot step in the machine. However, using $\theta_{r o t}$ as a constructive parameter of the three-dimensional simulation the effect of $\theta_{\text {rot }}$ on magnetic flux uniformity can be analyzed. It is possible to observe from Fig.12 that Carter's factor decrements asymptotically with twist angle. A possible explanation is that with greater twist angle more stator and 
rotor slots encounter in an alignment position and the distortion of the magnetic flux is compensated.

TABLE VIII

CARTER'S FACTOR COMPARISON EVALUATED ON THREE-DIMENSIONAL AIRGAP

\begin{tabular}{ccc}
\hline \hline Approach & Equation & $\boldsymbol{k}_{c}$ \\
& & 1.118 \\
Carter & $(1),(2)$ and (4) & 1.120 \\
Langsdorff & $(1),(3)$ and (4) & 1.090 \\
3D-FEM & $(8)$ & \\
\hline \hline
\end{tabular}

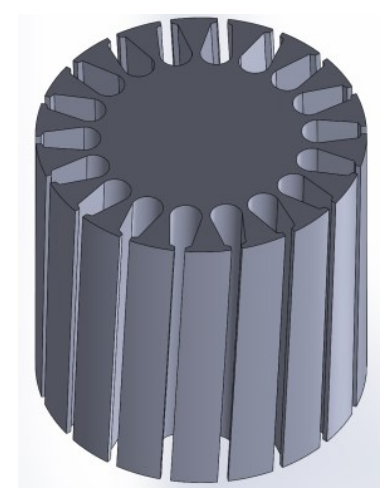

(a)

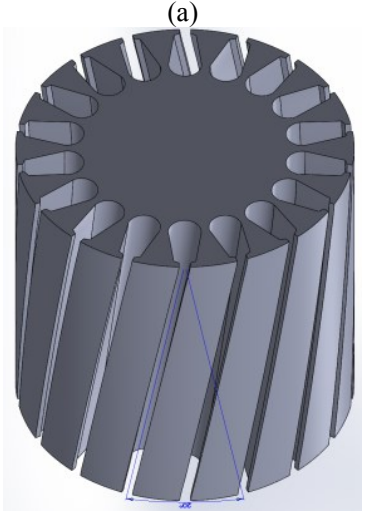

(b)

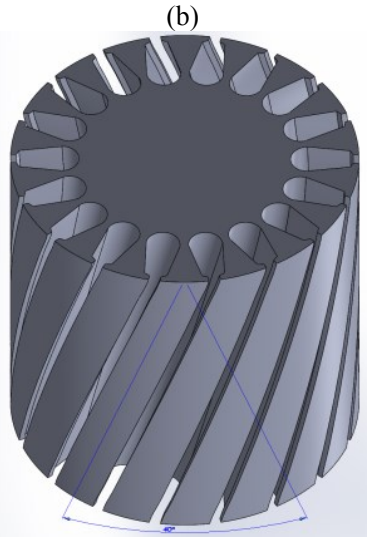

(c)

Fig. 11 Different twist angles in a rotor with 18 slots. (a) $5^{\circ}$, (b) $20^{\circ}$ and (c) $40^{\circ}$.

\section{CONCLUSIONS}

This paper presented a study of air gap geometry influence on the magnetic field flux density on rotating machines. Finite elements method was successfully used to measure uniformity of the magnetic flux distribution by calculating Carter's factor. Magnetostatic approach shows that slot constructive parameters type I can contribute in $2 \%$ for the sensitivity of the value of the Carter factor. Thus, even though $w_{\text {slot }}, h_{\max }$ and $w_{\max }$ are the most responsible for the magnetic flux uniformity on the air gap it is possible that search for optimized geometries has to take into consideration parameters intrinsically linked to the slot geometry.

Furthermore, slot geometry has great influence on magnetic equipotential midlines in doubled slotted air gaps. Deviations of $3 \%$ and $20 \%$ was observed, even on similar patterns of slots

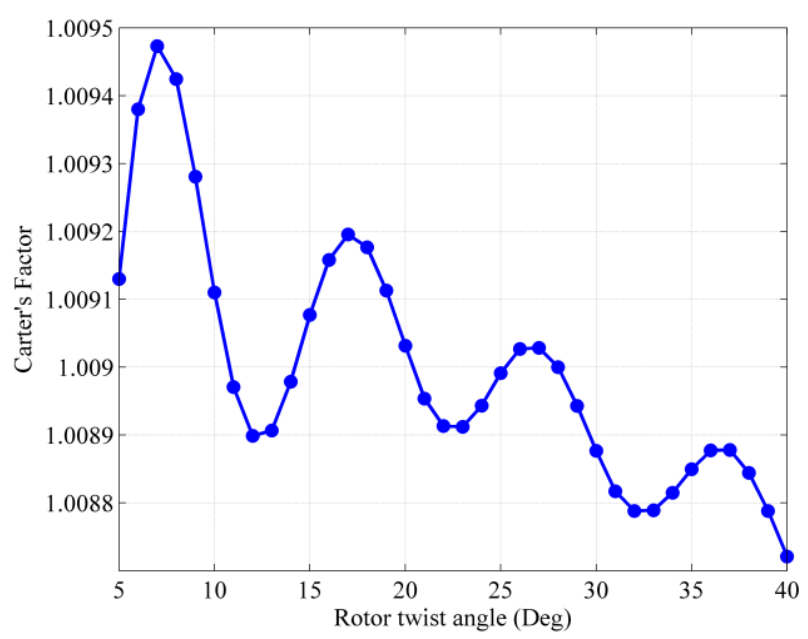

Fig.12 Graphic representation of the effect of twist angle $\theta_{\text {rot }}$ on Carter's factor.

on rotor and stator surfaces showing that the equipotential midline is not necessarily located in the middle of the gap length.

Additionally, genetic algorithm optimization process and Carter's factor calculation presented a slot geometry with an approximately uniform magnetic flux on air gap. Although real design machine process demands more restriction conditions on air gap constructive parameters the proposed optimization method is easily adapted including penalization factors to optimization problem and offers an efficient method to air gap optimization process.

Finally, the three-dimensional magnetic field mapping on the double air gap domain was performed to measure the Carter's factor. The proposed methodology calculates the Carter's factor using the intermediate magnetic scalar potential surface in air gap domain. The effect of the rotor twist angle was analyzed and show that Carter's factor decreases asymptotically with twist angle. Although the steady-state study performed in the simulation, the results represents the dynamic character of the magnetic flux uniformity in the machine, once the area of scalar magnetic equipotential surface remains constant over angular displacements of the rotor. 


\section{REFERENCES}

[1] V. H. Juha Pyrhönen, Tapani Jokinen, Design of rotating electrical machines. John Wiley \& Sons, Ltd, 2014.

[2] D. A. Lowther, "The Development of Industrially-Relevant Computational Electromagnetics Based Design Tools," IEEE Trans. on Magnetics, vol. 49, no. 5, pp. 2375-2380, May 2013.

[3] G. Kron, "Induction Motor Slot Combinations Rules to Predetermine Crawling, Vibration, Noise and Hooks in the Speed-Torque Curve," Transactions of the American Institute of Electrical Engineers, vol. 50, no. 2, pp. 757-767, June 1931.

[4] T. A. Lipo, Introduction to AC Machine Design. Wisconsin Power Electronics Research Center, Wisconsin, 2004.

[5] E. M. Freeman, "The calculation of harmonics, due to slotting, in the flux-density waveform of a dynamo-electric machine," in Proceedings of the IEE - Part C: Monographs, vol. 109, no. 16, pp. 581-588, September 1962.

[6] G. Liebmann, "The Change of Air-Gap Flux in Electrical Machines due to the Displacement of Opposed Slots," in Proceedings of the IEE - Part C: Monographs, vol. 104, no. 5, pp. 204-207, March 1957.

[7] F. W. Carter, "Corrigendum: The magnetic field of the dynamo-electric machine," in Electrical Engineers, Journal of the Institution of, vol. 65, no. 371, pp. 1025-, November 1927.

[8] F. W. Carter, "Note on air-gap and interpolar induction," in Electrical Engineers, Journal of the Institution of, vol. 29, no. 146, pp. 925-933, July 1900.

[9] F. W. Carter, "Air-gap induction", Electrical Word and Engineer, vol. 38, no. 22, pp. 884-888, November 1901.

[10] A. Langsdorf, Principles of Direct Current Machines. MacGraw-Hill: New York, 1959.

[11] H. VuXuan, D. Lahaye, H. Polinder and J. A. Ferreira, "Improved model for design of permanent magnet machines with concentrated windings," 2011 IEEE International Electric Machines \& Drives Conference (IEMDC), Niagara Falls, ON, 2011, pp. 948-954.

[12] H. Vu Xuan, D. Lahaye, H. Polinder and J. A. Ferreira, "Influence of stator slotting on the performance of permanent-magnet machines with concentrated windings," in IEEE Transactions on Magnetics, vol. 49, no. 2, pp. 929-938, Feb. 2013.

[13] W. P. Calixto, B. Alvarenga, A. P. Coimbra, A. J. Alves, L. Martins Neto, M. Wu, W. G. da Silva and E. Delbone, "Carter's fator calculation using domain transformations and the finite elemento method," International Journal of Numerical Modelling: Electronic Networks, Devices and Fields, vol. 25, n0. 3, pp. 236-247, 2012.

[14] A. C. Viorel, I. A. Viorel and L. Strete, "On the calculation of the Carter factor in the slotted electric machines," 2014 International Conference and Exposition on Electrical and Power Engineering (EPE), Iasi, 2014, pp. 332-336.

[15] Z. X. Fang, Z. Q. Zhu, L. J. Wu and Z. P. Xia, "Simple and accurate analytical estimation of slotting effect on magnet loss in fractional-slot surface-mounted PM machines," 2012 XXth International Conference on Electrical Machines, Marseille, 2012, pp. 464-470.

[16] W.P. Calixto, J. C. da Mota and B. P. Alvarenga, "Methodology for the reduction of parameters in the inverse transformation of Schwartz-Christoffel applied to electromagnetic devices with axial geometry", International Journal of Numerical Modelling, vol. 24, 2001.

[17] W. P. Calixto, E. G. Marra, L. da Cunha Brito and B. P. Alvarenga, "A new metthodology to calculate Carter fator using genetic algorithms," International Journal of Numerical Modelling, vol. 24, 2011.

[18] J.P.C. Kleijen, Experimental Design for Sensitivity Analysis, Optimization and Validation of Simulation Models, pp. 173-223, Johm Wiley \& Sons, Inc. 2007, [Online] Available: http://dx.doi.org/10.1002/ 9780470172445.ch6.

[19] K. L. Du and M. N. S. Swamy, Search and optimization by metaheuristics: techniques and algorithms inspired by nature, Birkhäuser, 2016 